\title{
Comparison of Patients' and Doctors' Reports on Health-related Quality of Life in Celiac Disease
}

\author{
${ }^{*}$ Sabine L. Vriezinga, ${ }^{*}$ Nora Farih, ${ }^{\dagger}$ Andrea E. van der Meulen-de Jong, ${ }^{\ddagger}$ Hein Putter,

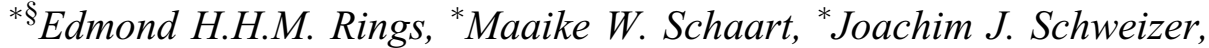 \\ ${ }^{*} \mid$ Margreet M.S. Wessels, and ${ }^{*}$ M. Luisa Mearin
}

\begin{abstract}
Objective: To investigate whether implementation of a celiac disease (CD)-specific health-related quality of life (HRQOL) questionnaire would add value to $\mathrm{CD}$ follow-up visits; we compared patients' self-reported CDspecific HRQOL with the physician's report provided during a regular CD follow-up visit in children and young adults.

Methods: A cross-sectional study in the control group of a study on selfmanagement in $\mathrm{CD}$ (CoelKids). Eligible patients had $\mathrm{CD}$ for $\geq 1$ year and were 25 years or younger. They completed a CD-specific HRQOL questionnaire (CDDUX) after their regular follow-up visit. Their physicians were unaware of the present study's objectives or selfreported HRQOL. Primary outcome: agreement between physicianreported and self-reported HRQOL. Secondary outcomes: patient variables predicting a discrepancy between reports, or a lower HRQOL. Results: Physician-reported HRQOL was available in 70 of 78 enrolled patients. The self-reported and physician-reported HRQOL were concordant in 30 of 70 ( $K=0.093), 6$ of them had a poor self-reported HRQOL. Reports were discrepant in 40 of 70 ; all 40 self-reported a poor HRQOL. Discrepancies occurred more frequently in patients with a disease duration $<9$ years $(32 / 40$ with discrepant reports were diagnosed $<9$ years ago vs $17 / 30$ with no discrepancy, $P<0.001)$ and in females $(35 / 40$ with discrepant reports were girls versus 16 of 30 with no discrepancy, $P=0.001)$. Both factors were predictors of a poorer HRQOL.

Conclusions: During regular CD follow-up visits, physicians did not report a poor HRQOL in 40 of 46 children and young adults with a poor selfreported HRQOL. This is consistent with previous studies examining other chronic diseases and supports the implementation of self-reported CDspecific HRQOL measurements in CD follow-up visits.
\end{abstract}

Key Words: CDDUX, CD-specific HRQOL, children, young adults

(JPGN 2017;64: 737-741)

eliac disease $(\mathrm{CD})$ is a chronic immune-mediated systemic disorder elicited by gluten in genetically susceptible individuals, affecting $1 \%$ to $3 \%$ of the general population $(1,2)$. The disease is treated with a life-long gluten-free diet (GFD) $(1,3)$.

Received January 2, 2016; accepted July 25, 2016.

From the * Department of Paediatrics, the tDepartment of Gastroenterology and Hepatology, the $\ddagger$ Department of Medical Statistics, Leiden University Medical Center, Leiden, the §Department of Paediatrics, Sophia Children's Hospital, Erasmus Medical Center, Rotterdam, and the IlDepartment of Paediatrics, Rijnstate Hospital, Arnhem, The Netherlands.

Address correspondence and reprint requests to Sabine L. Vriezinga, MD, Department of Paediatrics, Leiden University Medical Center, J6-S-208, 2333 ZA Leiden, The Netherlands (e-mail: s.l.vriezinga@lumc.nl).

Supplemental digital content is available for this article. Direct URL citations appear in the printed text, and links to the digital files are provided in the HTML text of this article on the journal's Web site (www.jpgn.org).

\section{What Is Known}

- Children and young adults with celiac disease have a self-reported neutral to low celiac disease-specific health-related quality of life.

- In patients with other chronic diseases, health-related quality of life is overestimated by their physician.

\section{What Is New}

- Physicians overestimate the health-related quality of life in children and young adults with celiac disease .

- Recently diagnosed and female patients are particularly vulnerable to overestimation.

- Our study supports the implementation of a selfreported celiac disease-specific health-related quality of life measurement in follow-up visits of celiac disease patients.

Standard medical care for CD patients consists of an evaluation of health status, weight, height (in children), GFD adherence, and CD-specific antibodies in serum (4). Health-related quality of life (HRQOL) is a more subjective and multidimensional concept containing physical, emotional, social, and cognitive domains that may vary over time and place $(5,6)$. Physicians need to be aware of their patients' HRQOL to intervene and facilitate improvement. In previous studies, untreated $\mathrm{CD}$ was associated with a reduced HRQOL, typically followed by an improvement after initiation of treatment $(7-11)$. Others found that the HRQOL in treated patients with $C D$ was still lower compared to that of the general population $(12,13)$, or significantly less in women with CD $(11,14-$ 16). The generic HRQOL instruments used in these studies allowed for comparison with normative data and across disease populations.

www.trialregister.nl registration number: NTR3688.

This study was funded by the Dutch "Maag Lever Darm Stichting" (WO1198), ZonMW (171201006), and STICOON (Dutch foundation for research in celiac disease). The sponsors had no role in the design and conduct of the study, collection, management, analysis, and interpretation of data or in preparation, review, and approval of the manuscript.

The authors have no conflicts of interest.

Copyright $(\mathcal{C} 2016$ by European Society for Pediatric Gastroenterology, Hepatology, and Nutrition and North American Society for Pediatric Gastroenterology, Hepatology, and Nutrition

DOI: $10.1097 / M P G .0000000000001355$ 
Disease-specific instruments may be more discriminating and sensitive to small differences and changes (17). In 2008, our research group designed and validated a CD-specific HRQOL questionnaire for children and young adults (CDDUX) which has then been used in research settings in different countries (17-19).

Although CD-specific HRQOL is an accepted outcome within a research environment, it is often not specifically measured during actual follow-up visits in clinical practice. Nevertheless, it has been established that in patients with other chronic diseases, the physician overestimates the patient's HRQOL (20-22). This has not yet been examined in CD. To investigate whether implementation of a CDspecific HRQOL questionnaire would add value to the follow-up visits for $\mathrm{CD}$, we compared the self-reported CD-specific HRQOL of a group of children and young adults with the physician's report provided during a regular outpatient follow-up visit for CD.

\section{METHODS}

In this cross-sectional study, we took advantage of the existing control group of the on-going multicenter research project in the Netherlands, called "CoelKids."

\section{CoelKids}

CoelKids aims to evaluate a multidisciplinary, internet-based, self-management system for CD-affected children and young adults ( $\leq 25$ years) to monitor their disease. $\mathrm{CD}$ was diagnosed according to the ESPGHAN criteria (3). The date of diagnosis was either the date when small bowel biopsies were performed and/or when positive CDspecific antibodies were determined. Patients were included in the study only if $\mathrm{CD}$ had been diagnosed for at least 1 year, and after informed consent was obtained. Exclusion criteria: IgA deficiency, lack of internet access, and/or difficulty in comprehending the Dutch questionnaires. Participants were randomized into 2 groups; the selfmanagement system group with online evaluations of their health, or the control group, wherein patients receive regular outpatient care for CD. The control group completed CD-specific HRQOL and GFD adherence questionnaires after their outpatient consultation. The study protocol was approved by the Medical Ethical Committee of the Leiden University Medical Center.

\section{Present Study}

We analyzed the HRQOL in the control group of CoelKids participants attending the outpatient clinic of the Leiden University Medical Center. The recruitment period was from May 2012 to August 2013. In order to accurately reflect the attention given to HRQOL by the physicians during a regular outpatient follow-up visit for $\mathrm{CD}$, the physicians received no instructions about how to conduct the HRQOL measurement. Furthermore, they were unaware of the study's objective and the patient's self-reported HRQOL. In "real life" physicians do not use a standardized method to assess HRQOL in patients with CD. Therefore, the physicianreported HRQOL was extracted from the clinical notes in the patient's Electronic Patient Record (EPR) by a well-instructed author who was blinded to the self-reported HRQOL. The physician-reported HRQOL was classified as: " 1 = good" if the physician reported there were no CD-associated problems (eg, "all is well, GFD accurately followed without problems"); " $2=$ bad" if CD-associated problems were reported; or " $0=$ not recorded" if the physician did not refer to the quality of life.

The self-reported HRQOL was measured online using the CDDUX, validated in Dutch children and adolescents (17). A proxy version for parents was also available. Thus, the self-reported HRQOL was either the patient's own response or his/her parent's. The CDDUX consists of 12 questions divided into 3 subscales: communication ( 3 items on feelings about explaining his/her disease), having $C D$ ( 3 items on feelings when s/he is offered gluten-containing food), and diet (6 items on feelings about dietary restrictions, lifelong aspects) (17). Response options were given on a 5-point Likert Scale ( $5=$ very bad; $1=$ very good) (17). The mean score was calculated to represent an overall evaluation (17). To compare the self-reported HRQOL with the physician's report, the self-reported HRQOL was dichotomized into "good" $\leq 3.00$; and "bad" $>3.00$. Reports were considered discrepant when the dichotomized self-reported HRQOL and the physician-reported HRQOL mismatched.

The patients' GFD adherence was assessed using the adapted Dutch version of a previously validated questionnaire, with scores ranging from 0 to 3 (23). Patients with scores of 0 to 1 were considered noncompliant. The remaining patients followed a GFD (either with errors [score 2] or without errors [score 3]). Patients not adhering to the GFD (score 0-1) were compared with the others (score 2-3).

\section{Outcomes}

The primary outcome was the agreement between the selfand physician-reported HRQOL. Secondary outcomes: the association between a discrepant self- and physician-reported HRQOL and the following patient variables: sex, patient age, age at diagnosis, years since diagnosis, GFD adherence, and whether or not HRQOL was parent-reported and the relation between the selfreported HRQOL and the aforementioned patient variables.

\section{Data Management and Statistical Analysis}

The participants entered their CDDUX responses into a Webbased data management application (NEN7510 certified). Data collected from the EPR were added manually. A Kappa value $(K)$ was computed to assess the agreement between the self- and physicianreported HRQOL. The $t$ test or chi-square test was used to determine the association between the aforementioned patient variables and a discrepant self- and physician-reported HRQOL. A univariate regression analysis was used to screen for patient variables that potentially influenced the self-reported HRQOL. Variables reaching borderline significance $(P<0.10)$ were selected for further evaluation in a multivariate model with a backward elimination approach. The $P$ value criteria for inclusion and exclusion were set at 0.05 and 0.10 , respectively. This method was repeated for the analysis of the HRQOL scores on the 3 subscales of the CDDUX (communication, having $C D$, and diet). Analyses were performed with SPSS software (version 20.0, IBM Corp., Armonk, NY).

\section{RESULTS}

\section{Patients}

The characteristics of the 78 patients are presented in Table 1. Twelve patients self-reported to be nonadherent to the GFD: 6 of them consumed normal quantities of gluten (score 0 ), the others made small dietary transgressions ( 5 regularly [score 0 ], 1 rarely [score 1]). Out of the 66 GFD adherent patients, 1 selfreported committing a dietary error (score 2). The parent-proxy version of the CDDUX questionnaire was used by 24 of 78 patients (mean age 7.8 years, range $2.2-13.3$ years). The outpatient consultations were performed by 6 different physicians.

\section{Health-related Quality of Life}

\section{Self-reported}

In general, the HRQOL of the 78 patients ranged from "neutral", to "bad," with a mean score of 3.29 on a scale of 
TABLE 1. Characteristics of the 78 patients with self-reported and physician-reported health related quality of life

\begin{tabular}{lc} 
Age, yr, mean (min-max) & $12.5(2.2-24.5)$ \\
Age $\geq 18$ y, N (\%) & $13(16.6)$ \\
Female, N (\%) & $57(73.1)$ \\
Age at diagnosis of CD, y, mean (min-max) & $5.3(1.0-23.4)$ \\
Duration of CD, y, mean (min-max) & $7.2(1.0-20.6)$ \\
Adherent to the GFD, $\mathrm{N}(\%)$ & $66(84.6)$ \\
\hline
\end{tabular}

$\mathrm{CD}=$ Celiac disease $; \mathrm{GFD}=$ gluten-free diet.

* GFD score $3(n=65)$ or $2(n=1)$ measured with the adapted Dutch version of a validated questionnaire (23).

$1-5(1=$ very good; $5=$ very bad $)$. Significantly better (lower) scores for the subscale communication than for having $C D$ or diet (Table 2) were observed. A "bad" or "very bad" HRQOL was reported by 42 patients. A "good" HRQOL was reported by 10 patients, whereas a "very good" HRQOL was reported by 1 . Male sex, older age, and longer disease duration were identified as predictors of a better HRQOL (univariate analysis, $P=0.007$; 0.042 and 0.010 respectively). Sex and disease duration together explained $16.7 \%$ of the variance in HRQOL (Table 3). The other patient variables did not influence the HRQOL. Predictors of a better score on the communication subscale were the male sex and using the parent-proxy questionnaire $(B=0.561, \mathrm{SE}=0.224,95 \%$ confidence interval $[\mathrm{CI}]=0.115-1.007, P=0.014$; and $B=0.430$, $\mathrm{SE}=0.215,95 \% \mathrm{CI}=0.002-0.859, P=0.049$ ), explaining $12.4 \%$ of the variance in the communication score. The only predictor of a better score on the having $C D$ subscale was a longer disease duration $(B=-0.046, \mathrm{SE}=0.015,95 \% \mathrm{CI}=-0.077$ to -0.015 , $P=0.004$ ), explaining $10.5 \%$ of the variance in scores on this subscale. A better score on the diet subscale was predicted by a longer disease duration and the male sex $(B=-0.038, \mathrm{SE}=0.018$, $95 \% \mathrm{CI}=-0.074$ to $-0.002, \quad P=0.039 ; \quad$ and $B=0.461$, $\mathrm{SE}=0.211, \quad 95 \% \quad \mathrm{CI}=0.040-0.882, \quad P=0.032), \quad$ explaining $11.4 \%$ of the variance in the score.

\section{Physician-reported}

The EPR of 70 of 78 patients contained information based on which the physician-reported HRQOL could be obtained. Six of 70 reports on HRQOL were interpreted as "bad" because the physician-detected problems resulting from the GFD. In the remaining 64 patients, no CD-related problems were identified; thus, the HRQOL was interpreted as "'good." Patient age, age at diagnosis, duration of disease, sex, and adherence to the GFD did not influence the physician-reported HRQOL $(P=0.137 ; 0.701 ; 0.302 ; 0.930$; 0.444 , respectively).

\section{Comparison of Self-reported and Physician- reported HRQOL}

The self-reported HRQOL was dichotomized into "good" for 24 patients and into "bad" for 46 patients. The physicianreported HRQOL matched the self-reported HRQOL in 30 of 70 cases $(K=0.093$, Table 4$)$. In the remaining 40 cases, a selfreported "bad" HRQOL was incongruously reported by the physician as being "'good." Discrepant reports of HRQOL were significantly more frequent in patients who had been diagnosed within the past 9 years $(32 / 40$ patients with discrepant reports diagnosed within past 9 years vs $17 / 30$ in whom the physician-reported HRQOL was correct, $P<0.001)$ and these patients were significantly more often female $(35 / 40$ patients with discrepant reports were girls vs $16 / 30$ in whom the physician-reported HRQOL was correct, $P=0.001$ ). Age, age at diagnosis of $\mathrm{CD}$, adherence to the GFD, and whether or not the HRQOL was assessed by parent-proxy was similarly distributed among both groups $(P=0.197 ; 0.899$; 0.394 , and 0.766 , respectively).

\section{DISCUSSION}

To the best of our knowledge, this is the first study comparing the CD patients' self-reported HRQOL against the physician's HRQOL reports during a regular outpatient follow-up visit for $\mathrm{CD}$. Our results indicate that there is an important discrepancy between these reports because in $57 \%$ of the patients, the physician had a different perception of the patients' HRQOL than the patient $\mathrm{him} / \mathrm{herself}$. What raises concern is that this occurred among patients considered to be especially vulnerable: those with a "bad"' self-reported HRQOL. Our data show that this problem occurs significantly more frequently in those who received the diagnosis within the past 9 years and in female patients, possibly due to their significantly poorer self-reported HRQOL, especially for the "communication" subscale, compared to their male counterparts $(P=0.014)$. On the other contrary, in patients with a "good" self-reported HRQOL, physicians correctly recognized it as being "good."

The female preponderance in our cohort $(73.1 \%)$ is a wellknown phenomenon in CD (1). Moreover, the neutral to bad selfreported HRQOL in our cohort is comparable with the results from previous studies in the Netherlands $(8,17)$. Therefore, our cohort may be considered as representative of the HRQOL of children and young adults with $\mathrm{CD}$ in our country. In addition, the improvement of the HRQOL as the disease duration becomes longer is supported

TABLE 2. Self-reported health-related quality of life ${ }^{*}$ in 78 children and young adults with celiac disease using the celiac disease-specific CDDUX questionnaire

\begin{tabular}{lccrr}
\hline HRQOL reported by & Total mean $(\mathrm{SD})$ & Communication mean $(\mathrm{SD})$ & Having CD mean $(\mathrm{SD})$ & Diet mean $(\mathrm{SD})$ \\
\hline All participants & $3.29(0.68)$ & $2.59^{\dagger}(0.92)$ & $3.62(0.74)$ & $3.48(0.87)$ \\
Patient $(\mathrm{N}=54)$ & $3.29(0.73)$ & $2.46^{\dagger}(0.92)$ & $3.64(0.79)$ & $3.52(0.92)$ \\
Parent-proxy $(\mathrm{N}=24)$ & $3.31(0.57)^{\ddagger}$ & $2.90^{\dagger, \ddagger}(0.88)$ & $3.57^{\ddagger}(0.63)$ & $3.39^{\ddagger}(0.74)$ \\
\hline
\end{tabular}

$\mathrm{CD}=$ celiac disease; HRQOL $=$ health-related quality of life; $\mathrm{SD}=$ standard deviation.

*A lower score indicating a better HRQOL.

†Significant difference between the mean score for "communication" and "having CD" and "communication" and "diet" (related-samples Wilcoxon signed rank test $P \leq 0.02$ ).

${ }^{\ddagger}$ No significant difference between the total mean HRQOL score reported by patients and parents, nor for the scores on the "having CD" and "diet" subscales (independent samples $t$ test $P=0.872,0.694,0.536$, respectively) borderline significance for the scores on the "communication" subscale $(P=0.048)$ 
TABLE 3. Predictors of the health-related quality of life in 78 children and young adults with celiac disease

\begin{tabular}{|c|c|c|c|c|c|c|c|c|}
\hline & \multicolumn{4}{|c|}{ Univariate analysis $^{*}$} & \multicolumn{4}{|c|}{ Multivariate analysis } \\
\hline & $B$ & SE & $95 \% \mathrm{CI}$ & $P$ & $B$ & SE & $95 \% \mathrm{CI}$ & $P$ \\
\hline Intercept & $\mathrm{NA}^{\dagger}$ & NA & NA & NA & 2.787 & 0.308 & 2.173 to 3.401 & $<0.001$ \\
\hline Age, y & -0.028 & 0.014 & -0.056 to -0.001 & 0.042 & & & & \\
\hline Age at diagnosis & 0.008 & 0.017 & -0.027 to 0.043 & 0.655 & & & & \\
\hline Duration of $\mathrm{CD}$ & -0.038 & 0.014 & -0.066 to -0.009 & 0.010 & -0.036 & 0.014 & -0.063 to -0.008 & 0.012 \\
\hline Female sex ${ }^{\ddagger}$ & 0.464 & 0.166 & 0.133 to 0.795 & 0.007 & 0.442 & 0.161 & 0.122 to 0.762 & 0.007 \\
\hline Voluntary gluten intake & -0.141 & 0.214 & -0.567 to 0.285 & 0.513 & & & & \\
\hline HRQOL reported by parent-proxy & 0.027 & 0.168 & -0.307 to 0.361 & 0.872 & & & & \\
\hline
\end{tabular}

$\mathrm{CD}=$ celiac disease; $\mathrm{CI}=$ confidence interval; HRQOL $=$ health-related quality of life; $\mathrm{SE}=$ standard error.

*By univariate analysis, patient's age, duration of CD, and sex were selected as potential predictors for the HRQOL. Using a multivariate model with a backward elimination approach, the age of the participant was eliminated as a factor influencing HRQOL.

${ }^{\dagger} \mathrm{NA}=$ not applicable because each univariate model has different intercepts.

${ }^{\ddagger}$ Male $=0$, female $=1$.

by a previous study (24). The lower HRQOL found in girls has been previously described in adults $(11,14-16)$, but not in children and/ or adolescents $(8,24,25)$. These studies did not use a validated CDspecific HRQOL questionnaire, but a generic questionnaire with $(14,25)$ or without $(11,15,16,24)$ added CD-specific questions. In our cohort, compliance with the GFD did not influence the selfassessed HRQOL, as was previously described in Dutch children whose HRQOL was evaluated in a research setting (7). Nevertheless, this should be interpreted with caution given that a relatively small fraction of patients in our cohort were noncompliant to the GFD (12/78). In contrast to our results, literature indicates that parents rate their child's HRQOL lower than the child does him/ herself $(17,18,24)$. Owing to the design of the larger research project the present study is part of, we only had either a patient or a parent report, not both. Excluding the 24 participants with parent-reported HRQOL gave results similar to those presented in this article. Furthermore, a recent study with the CDDUX in 214 Spanish children showed similar parent- and child-reported scores (19).

To address the discrepancy between the self-reported and physician-reported HRQOL in clinical practice, our results are consistent with previous studies on children affected by other chronic diseases (20-22). One meta-analysis has, in fact, demonstrated that the discrepancies between the physician- and childreported quality of life were mainly found in subjective rather than objective domains (20-22). The CD-specific HRQOL questionnaire CDDUX focuses on the subjective matters (feelings) (17). Previous studies do not report data on discrepancies between the physician- and self-reported HRQOL based on patient variables such as age, disease duration, and sex.

TABLE 4. Comparison of the self-reported and physician-reported health-related quality of life of $70^{*}$ children and young adults with celiac disease

\begin{tabular}{lccc}
\hline & \multicolumn{3}{c}{ Self-reported } \\
\cline { 2 - 4 } HRQOL & Good & Bad & Total \\
\hline Good & 24 & 40 & 64 \\
Bad & 0 & 6 & 6 \\
Total & 24 & 46 & 70 \\
\hline
\end{tabular}

HRQOL $=$ health-related quality of life.

* In 8 of 78 participants, the physician did not assess the HRQOL, selfreported HRQOL was good in $n=5$ and bad in $n=3$.
The strength of the present study is the use of a previously validated CD-specific HRQOL questionnaire. Moreover, the physicians were not informed about the study's objectives. Thus, a training effect was excluded, accurately reflecting the attention given to the HRQOL at a regular CD follow-up encounter. Indeed, it may be questioned whether each participating physician understood the concept of HRQOL similarly, as they did not use a standardized method to assess HRQOL. The physician's evaluation could have been obtained prospectively in a standardized manner. Our aim was, however, to compare the patient's self-reported HRQOL with the report provided by the physician during regular follow-up. Because the latter is typically done without using a standardized method, a questionnaire for physician-reported HRQOL would not provide us with an accurate reflection of the attention physicians give to this topic. In addition, assessing whether the outcome of a physiciancompleted HRQOL questionnaire agrees with the real (patientreported) HRQOL would not be of much use, as insufficient time during regular follow-up visits would prevent the physician from completing such a questionnaire. Consequently, to investigate whether or not the physician's understanding of the patient's HRQOL is accurate we extracted the physician-reported HRQOL from his/her clinical notes. One may argue that clinical notes do not always reflect the physician's understanding of a patient's HRQOL. Even if this were true, the lack of "poor" HRQOL documentation makes subsequent evaluations difficult and means that measures to increase the HRQOL are not being initiated. Our study included all physicians from our institution taking care of celiac children and young adults. This may have introduced interobserver variability. This is, however, a good reflection of real life: Some physicians may see more celiac patients than others and some may be more focused on HRQOL than others. Nevertheless, we found that the number of discrepancies was in fact neither influenced by whether the participant was assessed by a pediatric or adult gastroenterologist (physician- and self-assessed HRQOL matched in 26/63 participants assessed by a pediatrician vs in $4 / 7$ participants assessed by an adult gastroenterologist, $P=0.42$ ) nor by which physician assessed the patient $(P=0.67$, see Supplemental Digital Content, Table, http://links.lww.com/MPG/A747).

Our decision to include a self-reported CD-specific HRQOL score of exactly 3.00 in the category with a "good" score may be somewhat artificial. Including a score of $3.00(n=2)$ into the "bad" category, however, gave results similar to those presented in this article, and when all participants with a "neutral" score (2.61-3.40, N = 24) were excluded from our analysis (17). Measuring the HRQOL in a clinical setting may generate the expectation 
that the physician can improve the HRQOL (6). To achieve this in $\mathrm{CD}$ is challenging, firstly because the GFD is the only effective treatment for $\mathrm{CD}$, and secondly because of the variety of factors, which affect the generic HRQOL in chronic illness. To minimize the negative effect of a GFD and to maximize dietary adherence, it is, however, important that the physician is aware of the HRQOL of his/her patient. Patients may benefit from a consultation with a nutritionist and/or psychologist, or associating with peer-groups. It is possible that the results on the self-reported HRQOL vary in different countries. For example, Dutch children and adolescents with CD who both have a high general HRQOL, experienced a low to neutral CD-specific HRQOL (17). In contrast, similarly affected Argentine and Spanish patients had good and neutral CD-specific HRQOL scores, respectively $(18,19)$. Nevertheless, the Argentine patients indicated that the CDDUX questionnaire helped them express difficulties during the physician visit that otherwise would not have been discussed. Furthermore, their physicians indicated that the CDDUX helped them detect aspects that required action, for example, the need to refer to a nutritionist or psychologist (18).

In conclusion, there is a clinically significant discrepancy between the self-reported and physician-reported HRQOL in CDaffected children and young adults with a poor self-assessed HRQOL. Female patients and patients with a more recent diagnosis more often had these discrepant reports. Our study supports the implementation of a self-reported CD-specific HRQOL measurement in the clinical follow-up of the patients.

As the standard consultation time allotted for follow-up CD visits is limited, we suggest using a validated CD-specific HRQOL questionnaire before physician appointments. Sharing the results of the questionnaire may improve the patient/parent-doctor communication and the physicians' understanding of the needs and priorities of children and young adults with $\mathrm{CD}$.

Acknowledgments: The authors are grateful to all the patients and their parents for their efforts of taking part in the CoelKids study and to Dr D.C. Amado, visiting pediatrician at Leiden University Medical Center, for the English language editing.

\section{REFERENCES}

1. Mearin ML. Celiac disease among children and adolescents. Curr Probl Pediatr Adolesc Health Care 2007;37:86-105.

2. Myleus A, Ivarsson A, Webb C, et al. Celiac disease revealed in $3 \%$ of Swedish 12-year-olds born during an epidemic. J Pediatr Gastroenterol Nutr 2009;49:170-6.

3. Husby S, Koletzko S, Korponay-Szabo IR, et al. European Society for Pediatric Gastroenterology, Hepatology, and Nutrition guidelines for the diagnosis of coeliac disease. J Pediatr Gastroenterol Nutr 2012; $54: 136-60$.

4. Richtlijn Coeliakie en Dermatitis HerpetiformisHaarlem: Nederlandse Vereniging voor Maag-Darm-Leverartsen. [Dutch Guideline on Celiac Disease and Dermatitis Herpetiformis - Dutch Society of Gastroenterologists and Hepatologists, Haarlem: the Netherlands] 2008.

5. Guyatt GH, Feeny DH, Patrick DL. Measuring health-related quality of life. Ann Intern Med 1993;118:622-9.
6. Higginson IJ, Carr AJ. Measuring quality of life: using quality of life measures in the clinical setting. BMJ 2001;322:1297-300.

7. Hopman EG, Koopman HM, Wit JM, et al. Dietary compliance and health-related quality of life in patients with coeliac disease. Eur $J$ Gastroenterol Hepatol 2009;21:1056-61.

8. Kolsteren MM, Koopman HM, Schalekamp G, et al. Health-related quality of life in children with celiac disease. J Pediatr 2001;138:593-5.

9. Myleus A, Petersen S, Carlsson A, et al. Health-related quality of life is not impaired in children with undetected as well as diagnosed celiac disease: a large population based cross-sectional study. BMC Public Health 2014;14:425.

10. Nordyke K, Norstrom F, Lindholm L, et al. Health-related quality of life in adolescents with screening-detected celiac disease, before and one year after diagnosis and initiation of gluten-free diet, a prospective nested case-referent study. BMC Public Health 2013;13:142.

11. Tontini GE, Rondonotti E, Saladino V, et al. Impact of gluten withdrawal on health-related quality of life in celiac subjects: an observational casecontrol study. Digestion 2010;82:221-8.

12. Hauser W, Gold J, Stein J, et al. Health-related quality of life in adult coeliac disease in Germany: results of a national survey. Eur J Gastroenterol Hepatol 2006;18:747-54.

13. O'Leary C, Wieneke P, Buckley S, et al. Celiac disease and irritable bowel-type symptoms. Am J Gastroenterol 2002;97:1463-7.

14. Cranney A, Zarkadas M, Graham ID, et al. The Canadian Celiac Health Survey. Dig Dis Sci 2007;52:1087-95.

15. Roos S, Karner A, Hallert C. Psychological well-being of adult coeliac patients treated for 10 years. Dig Liver Dis 2006;38:177-80.

16. Casellas F, Rodrigo L, Vivancos JL, et al. Factors that impact healthrelated quality of life in adults with celiac disease: a multicenter study. World J Gastroenterol 2008;14:46-52.

17. Van Doorn RK, Winkler LM, Zwinderman KH, et al. CDDUX: a disease-specific health-related quality-of-life questionnaire for children with celiac disease. J Pediatr Gastroenterol Nutr 2008;47:147-52.

18. Pico M, Spirito MF. Implementation of a health-related quality of life questionnaire for children and adolescents with celiac disease. Arch Argent Pediatr 2014;112:19-25.

19. Torres JB, Roman E, Cilleruelo M, et al. Health-related quality of life in Spanish children with coeliac disease. J Pediatr Gastroenterol Nutr 2016;62:603-8

20. Janse AJ, Gemke RJ, Uiterwaal CS, et al. Quality of life: patients and doctors don't always agree: a meta-analysis. J Clin Epidemiol 2004;57:653-61.

21. Janse AJ, Sinnema G, Uiterwaal CS, et al. Quality of life in chronic illness: children, parents and paediatricians have different, but stable perceptions. Acta Paediatr 2008;97:1118-24.

22. Morrow AM, Hayen A, Quine S, et al. A comparison of doctors', parents' and children's reports of health states and health-related quality of life in children with chronic conditions. Child Care Health Dev 2012;38:186-95.

23. Biagi F, Bianchi PI, Marchese A, et al. A score that verifies adherence to a gluten-free diet: a cross-sectional, multicentre validation in real clinical life. Br J Nutr 2012;108:1884-8.

24. Bystrom IM, Hollen E, Falth-Magnusson K, et al. Health-related quality of life in children and adolescents with celiac disease: from the perspectives of children and parents. Gastroenterol Res Pract 2012;2012:986475.

25. Altobelli E, Paduano R, Gentile T, et al. Health-related quality of life in children and adolescents with celiac disease: survey of a population from central Italy. Health Qual Life Outcomes 2013;11:204. 